Related content

\section{Structural and luminescence investigation on gadolinium gallium garnet nanocrystalline powders prepared by solution combustion synthesis}

To cite this article: R Krsmanovi et al 2007 Nanotechnology 18325604

View the article online for updates and enhancements.
Nanocrystalline lanthanide-doped Lu3Ga5012 garnets: interesting materials forliaht-emitting devices
V Venkatramu, M Giarola, G Mariotto et al.
- Structural characterization and
luminescence properties of nanostructured lanthanide-dopedSc2O3 prepared by

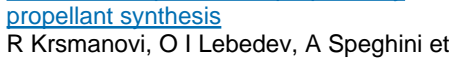 al.
A spectroscopic investigation of trivalent lanthanide dopedY2O3 nanocrystals
Fiorenzo Vetrone, John-Christopher Boyer, John A Capobianco et al.

\footnotetext{
Recent citations

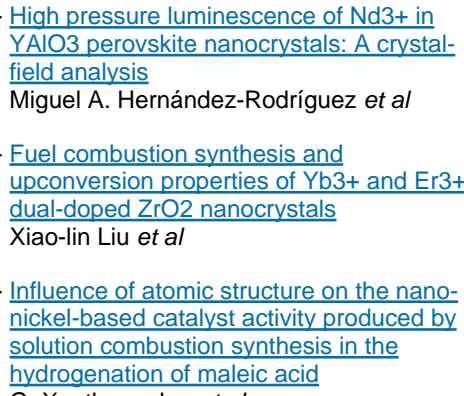

Hiah pressure luminescence of $\mathrm{Nd} 3+$ in YAIO3 perovskite nanocrystals: A crystalfield analysis

Miguel A. Hernández-Rodríguez et al

Fuel combustion synthesis and

upconversion properties of $\mathrm{Yb3}+$ and $\mathrm{Er} 3+$ dual-doped $\mathrm{ZrO} 2$ nanocrystals

Xiao-lin Liu et al

Influence of atomic structure on the nanonickel-based catalyst activity produced by solution combustion synthesis in the

hydrogenation of maleic acid

G. Xanthopoulou et al
} 


\title{
Structural and luminescence investigation on gadolinium gallium garnet nanocrystalline powders prepared by solution combustion synthesis
}

\author{
R Krsmanović ${ }^{1,5}$, V A Morozov ${ }^{2}$, O I Lebedev ${ }^{1}$, S Polizzi ${ }^{3}$, \\ A Speghini ${ }^{4}$, M Bettinelli ${ }^{4}$ and G Van Tendeloo ${ }^{1}$ \\ ${ }^{1}$ EMAT, University of Antwerp, Groenenborgerlaan 171, B-2020 Antwerp, Belgium \\ ${ }^{2}$ Department of Chemistry, Moscow State University, 119899 Moscow, Russia \\ ${ }^{3}$ Department of Physical Chemistry, Ca' Foscari University of Venice, Via Torino 155/b, \\ I-30172 Venice, Italy \\ ${ }^{4}$ Dipartimento Scientifico e Tecnologico, University of Verona and INSTM, UdR Verona, \\ Ca’ Vignal, Strada Le Grazie 15, I-37134 Verona, Italy \\ E-mail: radenka@vin.bg.ac.yu
}

Received 12 March 2007, in final form 30 May 2007

Published 13 July 2007

Online at stacks.iop.org/Nano/18/325604

\begin{abstract}
Nanocrystalline powders of undoped and lanthanide $\left(\mathrm{Pr}^{3+}, \mathrm{Tm}^{3+}\right)$-doped gadolinium gallium garnet, $\mathrm{Gd}_{3} \mathrm{Ga}_{5} \mathrm{O}_{12}$ (GGG), were prepared by propellant synthesis and studied by x-ray powder diffraction (XRD), electron diffraction (ED), high-resolution electron microscopy (HREM) and luminescence spectroscopy. The X-ray diffraction patterns of the GGG samples were analysed using the Rietveld method. The Rietveld refinement reveals the existence of two garnet-type phases: both are cubic (space group $I a \overline{3} d$ ) with a slightly different lattice parameter and probably a slightly different composition. Electron diffraction and electron microscopy measurements confirm the x-ray diffraction results. EDX measurements for lanthanide-doped samples show that stable solid solutions with composition $\mathrm{Gd}_{3-x} \mathrm{Ln}_{x} \mathrm{Ga}_{5} \mathrm{O}_{12}, x \approx 0.3(\mathrm{Ln}=\mathrm{Pr}$; Tm) have been obtained. The luminescence properties of the $\mathrm{Tm}^{3+}$-doped nanocrystalline GGG samples were measured and analysed.
\end{abstract}

\section{Introduction}

Lanthanide-doped gadolinium gallium oxide of composition $\mathrm{Gd}_{3} \mathrm{Ga}_{5} \mathrm{O}_{12}$ (GGG) with a garnet-type $\left(\mathrm{Ca}_{3} \mathrm{Al}_{2}\left(\mathrm{SiO}_{4}\right)_{3}\right.$ [1]) structure is an important optical material. Compounds with a garnet-type structure usually crystallize in a cubic symmetry (space group $\mathrm{O}_{\mathrm{h}}^{10}=I a \overline{3} d$ ). Nevertheless, possible distortions of the garnet structure are known $[2,3]$. The $\mathrm{Ca}_{3} \mathrm{Al}_{2}\left(\mathrm{SiO}_{4}\right)_{3}$ garnet structure is made up of $\left[\mathrm{CaO}_{8}\right]$ polyhedra, $\left[\mathrm{AlO}_{6}\right]$ octahedra and $\left[\mathrm{SiO}_{4}\right]$ tetrahedra. The constituent rare-earth (RE) elements in $\mathrm{RE}_{3} \mathrm{Ga}_{5} \mathrm{O}_{12}$ garnets occupy the Ca positions in the $\mathrm{Ca}_{3} \mathrm{Al}_{2}\left(\mathrm{SiO}_{4}\right)_{3}$ structure while the gallium cations are

\footnotetext{
5 Present address: Laboratory for Radiation Chemistry and Physics, Institute
} of Nuclear Sciences 'Vinca', PO Box 522, 11001 Belgrade, Serbia. located in octahedral and tetrahedral sites. The crystal growth of GGG, carried out with the Czochralski method, produces excellent colourless single crystals, with a high melting point $\left(1750^{\circ} \mathrm{C}\right.$ ) and a density of $7.08 \mathrm{~g} \mathrm{~cm}^{-3}$. The GGG lattice is body-centred cubic (bcc) with space group $\mathrm{O}_{\mathrm{h}}^{10}=I a \overline{3} d$ and a lattice parameter of $\approx 1.24 \mathrm{~nm}$. It has a high bulk refractive index $(n=2)$ and a transparency range from 400 to $7000 \mathrm{~nm}$. It is also a very good host for doping with rareearth ions. All these properties make doped or undoped GGG crystals very suitable for numerous technological applications, for instance as substrates for yttrium iron garnet (YIG) and YIG-like magneto-optical films [4-6] and as materials for solid-state lasers [7, 8].

Nanocrystalline GGG doped with rare earths is also an important luminescent material. It belongs to the group of 
oxide phosphors that are optimal for field-emission display (FED) and plasma panel display (PDP) devices [9-11]. As the demands for new displays with better resolution and increased luminous efficiency are high, the display industry needs efficient nanocrystalline luminescent materials. The new phosphors have to be thermally stable, highly luminescent, radiation resistant and single phase with a small particle size. Such materials are also very efficient in infrared (IR)-to-visible up-conversion with possible applications as IR-sensitive phosphors, biological labels and up-converting materials. The appearance of many different synthesis techniques like pulsed laser deposition [12], solution combustion (propellant) [13-17], sol-gel [18], sol-gel combustion [19], wet chemical [20] or co-precipitation [21, 22] synthesis enables the production of different nanophosphors with improved characteristics. One of the advantages of these techniques is that nanocrystalline powders are obtained as a final product. Hence there is no need to use grinding or milling processes, used conventionally for the commercial phosphors with larger, micrometre-size particles. Such mechanical treatments are known to introduce surface defects and add impurities. Surface defects might cause non-radiative relaxations of the excited states and thus drastically decrease the luminescence efficiency of the phosphor. The luminescence efficiency will also decrease if dopant ions are concentrated on the nanocrystal surface or on the grain boundaries [9].

The luminescence properties of GGG single crystals doped with trivalent lanthanide ions have been studied in detail [7, 8, 23-25]. In recent years it has been shown that nanocrystalline lanthanide-doped GGG samples, obtained by the propellant technique, have very interesting luminescence properties [26-29]. In particular, intense up-conversion luminescence in the visible region is observed upon excitation with IR radiation. Moreover, the up-conversion processes are more efficient in lanthanide-doped GGG than in $\mathrm{Y}_{2} \mathrm{O}_{3}$ or $\mathrm{Lu}_{2} \mathrm{O}_{3}$ prepared with the same synthesis technique. This was explained by a much lower concentration of adsorbed contaminants (carbonate and hydroxide ions) on the surface of the GGG nanocrystals $[26,27]$ with respect to $\mathrm{Y}_{2} \mathrm{O}_{3}$ or $\mathrm{Lu}_{2} \mathrm{O}_{3}$ nanocrystalline materials.

Even though it has been shown that microstructural parameters such as size and surface of the nanocrystals, level of agglomeration, activator concentration and presence of defects are responsible for the luminescence efficiency of the nanocrystalline phosphors [13, 14, 18, 30-33], only a few detailed structural characterizations have been reported [17, 22, 23, 34, 35]. In our previous work [36] we studied a series of GGG samples, obtained via solution combustion synthesis, doped with different lanthanide ions (Tm, Er, Ho, Eu, Sm, Nd, Pr) and with different concentrations $(1,5,10 \mathrm{~mol} \%)$. For all samples XRD analysis revealed the presence of the cubic GGG phase. Each peak of the cubic GGG phase was accompanied by a weak asymmetric peak shifted to lower $2 \theta$ values. The presence of these asymmetric peaks cannot be explained as an effect of microstrains, as their value is low (of the order of $10^{-3}$ ), but it is more likely attributed to the existence of a second phase. The regular appearance of the asymmetric peaks in all samples suggests that highly reproducible solid solutions of GGG are obtained with the propellant technique.
In this work a more detailed investigation on the structure, morphology and composition of undoped, and $\mathrm{Pr}^{3+}$ - or $\mathrm{Tm}^{3+}$ doped GGG nanocrystalline powders is presented, together with the luminescence properties for the $\mathrm{Tm}^{3+}$-doped GGG samples. The lanthanide ions can easily enter as dopants in the GGG lattice structure as these ions have nearly the same ionic size of the $\mathrm{Gd}^{3+}$ ions. The incorporation of $\mathrm{Ln}^{3+}$ ions in the GGG lattice has been addressed by some of us in a previous paper [37]. For a detailed structural characterization we focused on the two more concentrated doped samples, as the possible changes in the garnet structure should be more evident. The $\mathrm{Pr}^{3+}$ - and $\mathrm{Tm}^{3+}$-doped samples have been chosen as representative of the larger $\mathrm{Ln}^{3+}$ ions at the beginning of the lanthanide series and of the smaller ions at the end, respectively.

One of the aims of this investigation is to identify the phases and to determine the lattice parameters, crystallite dimensions and chemical composition for the nanocrystalline GGG samples. Such investigations were performed using xray powder diffraction, electron diffraction and high-resolution electron microscopy. The analysis of the x-ray diffraction patterns was made using the Rietveld method. Compositional measurements were done using energy dispersive x-ray (EDX) spectroscopy coupled to a scanning electron microscope or a transmission electron microscope. The spectroscopic properties of the $\mathrm{Tm}^{3+}$-doped GGG samples were studied using laser-excited luminescence spectroscopy. The optical spectroscopy of the $\mathrm{Pr}^{3+}$-doped samples will be the subject of a separate paper [38].

\section{Experimental details}

\subsection{Synthesis}

GGG nanocrystals, undoped and doped with $\mathrm{Pr}^{3+}$ and $\mathrm{Tm}^{3+}$ ions, were synthesized using a solution combustion procedure starting from an aqueous mixture of $\left(\mathrm{NH}_{2} \mathrm{NH}\right)_{2} \mathrm{CO}(98 \%)$, $\mathrm{Gd}\left(\mathrm{NO}_{3}\right)_{3} \cdot 6 \mathrm{H}_{2} \mathrm{O}(99.99 \%), \mathrm{Ga}\left(\mathrm{NO}_{3}\right) \cdot 3 \mathrm{H}_{2} \mathrm{O}(99.999 \%)$ and $\mathrm{Ln}\left(\mathrm{NO}_{3}\right)_{3} \cdot 6 \mathrm{H}_{2} \mathrm{O}(99.9 \%)(\mathrm{Ln}=\mathrm{Pr} ; \mathrm{Tm})$. GGG nanocrystalline samples containing $10 \%$ of $\mathrm{Pr}^{3+}$ ions and $1,5,10 \%$ of $\mathrm{Tm}^{3+}$ ions with respect to $\mathrm{Gd}^{3+}$ were prepared. Details of this preparation method can be found in $[13,14]$. After the combustion, the powders were annealed in air for $1 \mathrm{~h}$ at $500^{\circ} \mathrm{C}$ in order to decompose the residual carbohydrazide and nitrate ions. The samples were subsequently heat treated in air at $800^{\circ} \mathrm{C}$ for $72 \mathrm{~h}$.

\subsection{Structural characterization}

The morphology, local composition and microstructure of the GGG samples were studied by x-ray diffraction, electron microscopy and EDX analysis.

The X-ray diffraction (XRD) patterns were measured on a Thermo ARL X'TRA powder diffractometer $(\mathrm{Cu} \mathrm{K} \alpha$ radiation, $\lambda=1.5418 \AA$ A , Bragg-Brentano geometry, Peltier-cooled CCD detector). XRD data were collected at room temperature over the $10^{\circ}-100^{\circ} 2 \theta$ range with steps of $0.02^{\circ}$. XRD patterns were analysed by the Rietveld method [39, 40] using the RIETAN-98 program [41]. The fractional coordinates of GGG (space group $\mathrm{I} a \overline{3} d$ [42]) and $\mathrm{Ca}_{3}\left(\mathrm{Al}_{0.8} \mathrm{Fe}_{0.2}\right)_{2}\left(\mathrm{SiO}_{4}\right)_{3}$ (space group $F d d d[3]$ ) were used as initial parameters. The 
background, profile and unit-cell parameters were refined. Atomic coordinates and atomic displacement parameters were not refined.

Scanning electron microscopy (SEM) observations were performed with a Jeol JEM-5510 equipped with an INCA xray microanalysis unit. The composition of the samples was determined by $\mathrm{x}$-ray energy-dispersion spectrometry (EDS) acquiring a spectrum for $600 \mathrm{~s}$ (live time) at the accelerating voltage of $30 \mathrm{kV}$. For this analysis powders were cold-pressed into pellets of $3 \mathrm{~mm}$ diameter under a load of about 2 tonnes of pressure and then left uncoated.

Transmission electron microscopy (TEM) was used to obtain information about the structure and size distribution of the nanoparticles. Electron diffraction and EDX analysis were performed on a Philips CM20 microscope, operating at $200 \mathrm{kV}$ and equipped with a LINK-2000 EDX attachment. Highresolution electron microscopy (HREM) observations were made on a Jeol 4000EX electron microscope. TEM specimens were prepared by dispersing the finely crushed powdered sample in pure ethanol, exposing the solution to ultrasonic vibrations for about $10 \mathrm{~min}$ and then depositing a drop of solution on a holey carbon film grid. The CrystalKitX and MacTempas software packages (software for High Resolution Electron Microscopy, Total Resolution, 20 Florida Ave., Berkeley, CA, USA) were used to simulate electron diffraction patterns and HREM images.

\subsection{Luminescence spectroscopy}

The room-temperature emission spectra and decay curves of the $\mathrm{Tm}^{3+}$-doped GGG nanocrystalline samples were measured upon excitation with the third harmonic (at $355 \mathrm{~nm}$ ) of the fundamental radiation of a Quanta System SYL201 Nd-YAG pulsed laser. The emission radiation was dispersed with a Jobin Yvon HR450 half-metre monochromator equipped with a 150 lines $\mathrm{mm}^{-1}$ grating. A Jobin Yvon Spectra One aircooled $\mathrm{CCD}$ device was employed to record the emission spectra. A water-cooled Hamamatsu GaAs photomultiplier and a Le Croy Waverunner LT342 digital oscilloscope were used to obtain the emission decay curves.

\section{Results and discussion}

\subsection{Composition determination}

After synthesis, the GGG powders consist of agglomerates with an irregular shape and a size ranging from a few tens of microns to a few millimetres. Before observation in the electron microscope, the powders were lightly ground in a mortar to obtain smaller agglomerates.

The average composition of the undoped and $10 \% \mathrm{Pr}^{3+}$ and $\mathrm{Tm}^{3+}$-doped GGG samples was determined by energydispersive $\mathrm{x}$-ray (EDX) spectroscopy in a scanning electron microscope. The EDX spectra, shown in figure 1, confirm the lanthanide doping of the GGG samples and show that the elemental ratio $\mathrm{Gd} / \mathrm{Pr} \approx 8.6$ is close to that expected for a solid solution of $\mathrm{Gd}_{2.7} \mathrm{Pr}_{0.3} \mathrm{Ga}_{5} \mathrm{O}_{12}$ composition. For the $\mathrm{Tm}^{3+}$. doped sample it was more difficult to obtain accurate results by EDX, due to the overlap of some Tm and Gd peaks.

Using the EDX mapping technique it was also possible to verify the homogeneity of the samples. Elemental maps

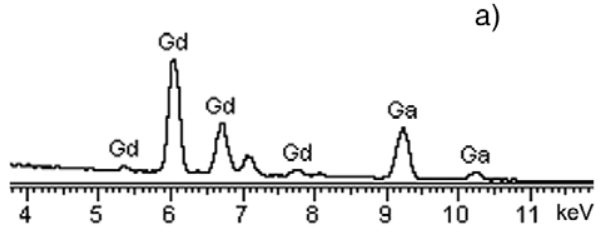

b)

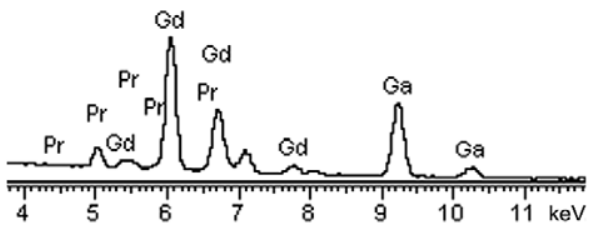

c)

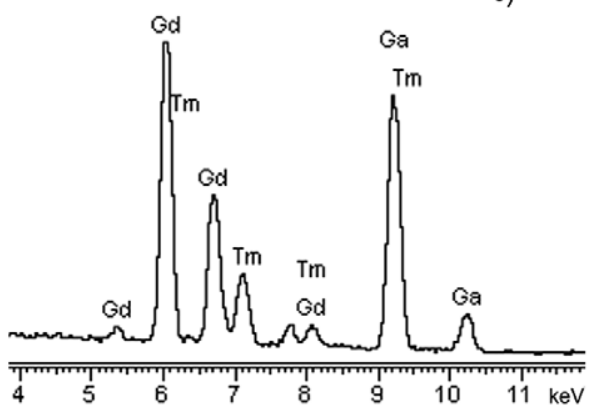

Figure 1. EDX spectra of undoped $\mathrm{Gd}_{3} \mathrm{Ga}_{5} \mathrm{O}_{12}$ (a) and $\mathrm{Gd}_{3} \mathrm{Ga}_{5} \mathrm{O}_{12}$ doped by lanthanides $\left(\operatorname{Pr}^{3+}(\mathrm{b}), \mathrm{Tm}^{3+}(\mathrm{c})\right)$.

of $\mathrm{Gd}, \mathrm{Ga}, \mathrm{O}$, Pr and Tm show uniform density, indicating good homogeneous distribution of praseodymium and thulium throughout the material.

EDX measurements performed in the TEM apparatus and using the classical composition determination software, show a $\mathrm{Ga} / \mathrm{Gd}$ ratio for the undoped GGG nanocrystalline sample of $1.44 \pm 0.06$ (the stoichiometric $\mathrm{Ga} / \mathrm{Gd}$ ratio is 1.67). The elemental analysis gives $59 \pm 2$ at. $\%$ for $\mathrm{Ga}$ and $41 \pm 2$ at.\% for Gd. These results slightly deviate from the stoichiometric 62.5 at.\% and 37.5 at.\% for $\mathrm{Ga}$ and $\mathrm{Gd}$, respectively. Therefore, the element contents in a commercial $\mathrm{Gd}_{3} \mathrm{Ga}_{5} \mathrm{O}_{12}$ sample (ALDRICH, 99.9\%) were determined as references. The experimental EDX results for the commercial GGG gave $61 \pm 1$ at. $\%$ for Ga and $39 \pm 1$ at. $\%$ for Gd, with a $\mathrm{Ga} / \mathrm{Gd}$ ratio of $1.56 \pm 0.03$. The element content values found for the commercial sample are closer to the ideal stoichiometry than for the nanocrystalline GGG samples, that appear to be slightly richer in Gd than predicted from the formula.

\subsection{X-ray diffraction study}

Parts of the XRD pattern from $10^{\circ}$ to $65^{\circ}$ and from $38^{\circ}$ to $48^{\circ}$ for undoped GGG are shown in figure 2. The first analysis of the XRD pattern for GGG by the Rietveld method was done in the model of a single-phase cubic garnet. As shown in figure 2(a), each reflection of the GGG cubic phase on the XRD patterns is accompanied by an asymmetric peak shifted to the 

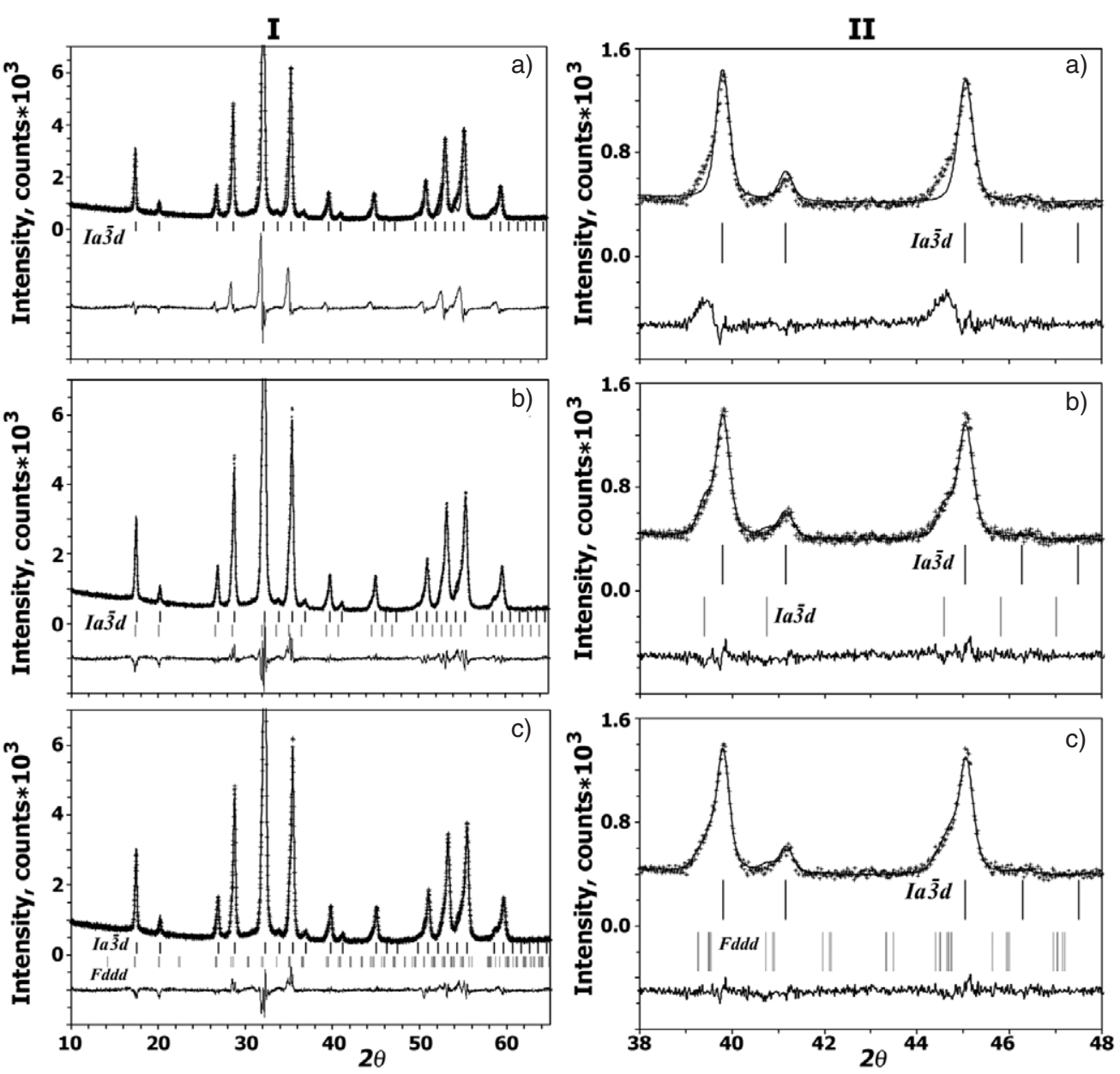

Figure 2. Parts (I-from $10^{\circ}$ to $65^{\circ}$ and II-from $38^{\circ}$ to $48^{\circ}$ ) of the observed (crosses), calculated (solid line) and difference XRD patterns for undoped $\mathrm{Gd}_{3} \mathrm{Ga}_{5} \mathrm{O}_{12}$ : (a) model with one single-phase cubic garnet; (b) model with two cubic phases; (c) model with one cubic phase and one orthorhombic distorted phase. Tick marks denote the peak positions of possible Bragg reflections of the different phases.

left of it. Rietveld difference patterns (figure 2(a)) show a clear evidence of the presence of a second garnet-type phase. There are two possible models for the two-phase refinement: (i) two cubic phases with a different unit-cell parameter; (ii) a cubic and a distorted garnet-type phase. The GGG lattice is bodycentred cubic (space group $I a \overline{3} d$ ) with a lattice parameter of $\approx 1.24 \mathrm{~nm}$. The formation of a second cubic phase with a larger unit cell than GGG might be explained in terms of the formation of a solid solution $\mathrm{Gd}_{3+x} \mathrm{Ga}_{5-x} \mathrm{O}_{12}$ with a statistical distribution of $\mathrm{Gd}^{3+}$ and $\mathrm{Ga}^{3+}$ on the octahedral site. It should be noted that a heat treatment at $1450{ }^{\circ} \mathrm{C}$ for $6 \mathrm{~h}$ of the GGG:1 mol\% Ho, with a second garnet-type phase, leads to the formation of a pure GGG cubic phase [36]. The fit for this model with the experimental data can be seen in figure 2(b). A slightly better agreement between the observed and the calculated patterns, however, occurs for a model with a cubic $(I a \overline{3} d)$ and an orthorhombic $(F d d d)$ garnet-type phase with unit-cell parameters $a_{\mathrm{o}} \approx a_{\mathrm{c}} \times \sqrt{2}, b_{\mathrm{o}} \approx a_{\mathrm{c}}, c_{\mathrm{o}} \approx$ $a_{\mathrm{c}} \times \sqrt{2}$, where $a_{\mathrm{o}}, b_{\mathrm{o}}, c_{\mathrm{o}}$ and $a_{\mathrm{c}}$ are the unit-cell parameters of orthorhombic and cubic phases (figure 2(c)), respectively. Such an orthorhombic phase might be metastable and could transform to the cubic garnet phase at higher temperatures.
Bazzoni et al [22] recently observed various metastable phases in nanocrystalline GGG prepared with the co-precipitation technique. The type of synthesis of the nanosized material and the post-synthesis treatments, such as annealing temperature or mechanical milling, can be responsible for the appearance of various metastable GGG phases. The experimental/refinement conditions, final $R$ factors, and lattice parameters for all models of the Rietveld refinement are summarized in table 1. A final decision between two cubic phases or a cubic and a distorted cubic phase cannot be made from the XRD data only. TEM will have to provide the answer (see below).

XRD patterns for the lanthanide $\left(\mathrm{Pr}^{3+}, \mathrm{Tm}^{3+}\right)$-doped GGG are very similar to the XRD pattern for undoped GGG. Each reflection of the GGG $10 \mathrm{~mol} \% \operatorname{Pr}(\mathrm{Tm})$ cubic phase in the XRD patterns is also characterized by an asymmetric peak. The unit-cell parameters of undoped GGG and GGG doped with the lanthanide ions after Rietveld refinement are given in table 2. The unit-cell parameter changes with the lanthanide doping and the volume of the unit cell increases for the Pr-doped sample and decreases for the Tm-doped sample. A similar dependence on the doping is observed for the unitcell parameter of the cubic phase and agrees with the size of the $\mathrm{Pr}^{3+}$ and $\mathrm{Tm}^{3+}$ ions with respect to the host $\mathrm{Gd}^{3+}$ cations. 
Table 1. Crystallographic data, experimental conditions, and refinement results for undoped $\mathrm{Gd}_{3} \mathrm{Ga}_{5} \mathrm{O}_{12}$.

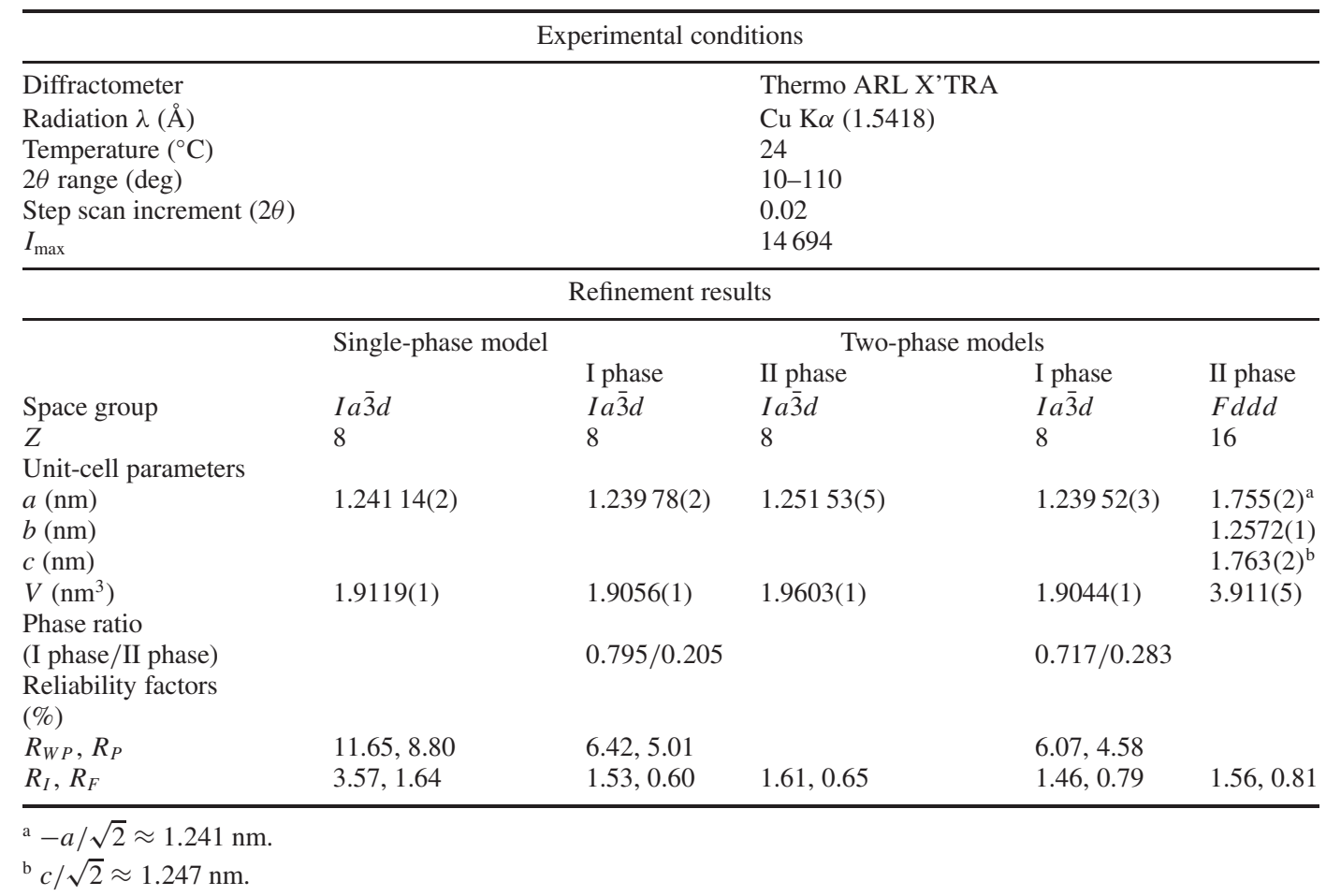

Table 2. Unit-cell parameters for $\mathrm{Gd}_{3} \mathrm{Ga}_{5} \mathrm{O}_{12}$ and $\mathrm{Gd}_{3} \mathrm{Ga}_{5} \mathrm{O}_{12}$ doped by lanthanide ( $\mathrm{Pr}, \mathrm{Tm}$ ) (space groups $I a \overline{3} d$ and $F d d d$ ) after Rietveld refinements.

\begin{tabular}{lllllll}
\hline Compound & $r_{\mathrm{VIII}}(\mathrm{Ln}),(\AA)[53]$ & Model & $a(\mathrm{~nm})$ & $b(\mathrm{~nm})$ & $c(\mathrm{~nm})$ & $V\left(\mathrm{~nm}^{3}\right)$ \\
\hline GGG & 1.06 & 1 phase & $1.24114(2)$ & & & $1.9119(1)$ \\
& & 2 phases & $1.23952(3)$ & & & $1.9044(1)$ \\
& & $1.755(2)$ & $1.2572(1)$ & $1.763(2)$ & $3.911(5)$ \\
GGG & \multirow{2}{*}{1.14} & 1 phase & $1.24302(6)$ & & & $1.9206(2)$ \\
$10 \mathrm{~mol} \% \mathrm{Pr}$ & & 2 phases & $1.24148(4)$ & & & $1.9134(1)$ \\
& & & $1.7664(3)$ & $1.2473(3)$ & $1.7856(2)$ & $3.934(1)$ \\
GGG & 0.99 & 1 phase & $1.23763(5)$ & & & $1.8957(1)$ \\
$10 \mathrm{~mol} \% \mathrm{Tm}$ & & 2 phases & $1.23722(2)$ & & & $1.8938(1)$ \\
& & & $1.7608(2)$ & $1.2439(2)$ & $1.7785(2)$ & $3.895(1)$ \\
\hline
\end{tabular}

Table 3. Results of EDX quantitative analysis in the transmission electron microscope for undoped $\mathrm{Gd}_{3} \mathrm{Ga}_{5} \mathrm{O}_{12}$ and $\mathrm{Gd}_{3} \mathrm{Ga}_{5} \mathrm{O}_{12}$ doped with $10 \mathrm{~mol} \% \mathrm{Pr}$ and $\mathrm{Tm}$. Average values with errors estimated as one standard deviation are reported.

\begin{tabular}{llllll}
\hline at.\% sample & $\mathrm{Ga} \mathrm{K}$ & $\mathrm{Gd} \mathrm{L}$ & $\mathrm{PrL}$ & $\mathrm{TmL}$ & $x$ \\
\hline $\mathrm{Gd}_{3-x} \mathrm{Pr}_{x} \mathrm{Ga}_{5} \mathrm{O}_{12}$ & $59 \pm 2$ & $37 \pm 2$ & $4.2 \pm 0.5$ & & $0.31 \pm 0.05$ \\
$\mathrm{Gd}_{3-x} \mathrm{Tm}_{x} \mathrm{Ga}_{5} \mathrm{O}_{12}$ & $59 \pm 2$ & $37 \pm 2$ & $4.3 \pm 0.5$ & $0.31 \pm 0.04$ \\
$\mathrm{Gd}_{3} \mathrm{Ga}_{5} \mathrm{O}_{12}$ & $59 \pm 2$ & $41 \pm 2$ & & \\
\hline
\end{tabular}

\subsection{Electron microscopy study}

The elemental composition of undoped GGG and lanthanidedoped GGG was confirmed by EDX analysis in the transmission electron microscope on different crystallites; at the same time, the structure was verified by electron diffraction. The EDX analysis was performed with the Ga K, Gd L, Pr L and Tm L lines, taking measurements for at least 25 different crystallites for each sample. The results of the EDX quantitative analysis for undoped and doped $\mathrm{Gd}_{3} \mathrm{Ga}_{5} \mathrm{O}_{12}$ samples are listed in table 3.
TEM observations show that agglomerates are composed of nanosized crystallites with an irregular form and various dimensions, ranging from 30 to $100 \mathrm{~nm}$ (figure 3 ). The voids present are the result of the very rapid combustion reaction during synthesis.

The cubic structure of $\mathrm{Gd}_{3} \mathrm{Ga}_{5} \mathrm{O}_{12}$ was confirmed for all samples by analysing the electron diffraction (ED) patterns. The ring pattern (inset in figure 3 ) indicates that our material is polycrystalline. The grainy rings are related to the fact that the constituent crystallites have a size of $30 \mathrm{~nm}$ or more. The ring pattern could be indexed as the cubic $\mathrm{Gd}_{3} \mathrm{Ga}_{5} \mathrm{O}_{12}$ structure: in figure 3 , for clarity, only the most intense rings are indexed.

The local crystal structure was investigated by analysing single-domain ED patterns and the corresponding highresolution images. The model used for the image simulations is the $\mathrm{Gd}_{3} \mathrm{Ga}_{5} \mathrm{O}_{12}$ structure with space group $\mathrm{O}_{\mathrm{h}}^{10}(I a \overline{3} d)$ and $a \approx$ $1.24 \mathrm{~nm}$. Single-domain ED patterns obtained for the main zones, $[001]^{*},[0 \overline{1} 1]^{*}$ and $[11 \overline{1}]^{*}$, are shown in figure 4 . All ED patterns are completely indexed on a cubic unit cell with lattice parameter around $1.24 \mathrm{~nm}$, in agreement with the data 


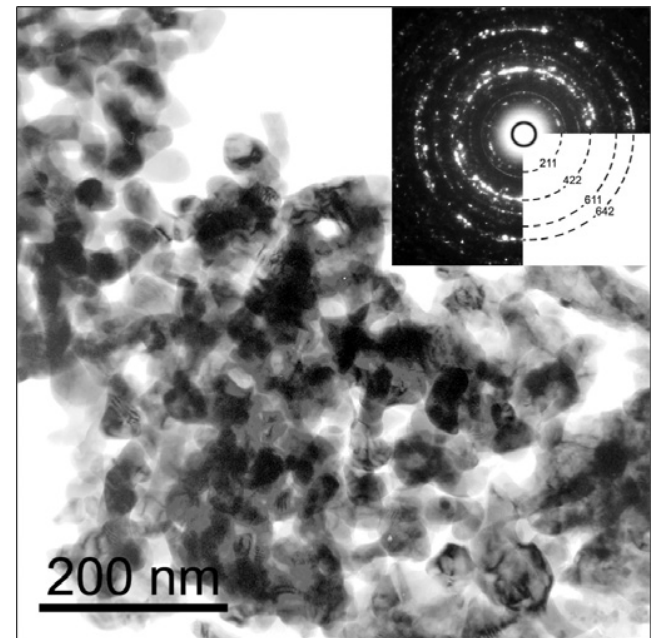

Figure 3. TEM image and corresponding electron diffraction ring pattern of undoped $\mathrm{Gd}_{3} \mathrm{Ga}_{5} \mathrm{O}_{12}$. Only the most intense rings are indexed.

from x-ray powder diffraction (table 2). Using TEM, however we could not observe any evidence for an orthorhombic garnettype phase as eventually suggested from the x-ray diffraction analysis. No spot splitting, and no superlattice reflections were present in any of the patterns. We did, however, notice a slight variability in the lattice parameter between different grains.

The general reflection conditions for the space group $I a \overline{3} d$ are as follows. $h k l: h+k+l=2 n, 0 k l: k, l=2 n, h h l$ : $2 h+l=4 n, h 00: h=4 n$, where $h, k$ and $l$ are permutable. ED patterns of [001]* and [111 $]^{*}$ zones exhibit reflections with indices $h 00 \neq 4 n$ (200) and $h h l, 2 h+l \neq 4 n$ (110) due to double diffraction. For the $[0 \overline{1} 1]^{*}$ zone these extra reflection do not occur, as double diffraction conditions are not present.

In order to check the quality of the nanocrystals, we analysed a number of them with HREM. The nanocrystals proved to be stable under the electron beam. However, we worked very fast, using the smallest beam intensity, and controlling any possibly e-beam transformation (or damage) by taking the series of images and comparing the first and the last image. In figure 5, an HREM image of one Pr-doped GGG nanocrystal along the [001]* direction is shown. The surface is free of defects and without any amorphous layer. The structure can be interpreted in terms of cation and oxygen columns where brighter dots correspond to $\mathrm{Gd}$ and $\mathrm{Ga}$ atomic columns (and/or Pr). Computer simulations made for the trial model, in which dopant ions of $\mathrm{Pr}^{3+}$ enter the structure by replacing $\mathrm{Gd}^{3+}$ ions randomly, match the experimental HREM images well. No differences in intensity are observed, in agreement with the fact that the Pr doping is homogeneous. This is also consistent with the absence of diffuse intensity in the ED patterns. No cation ordering is observed, but EDX analysis in the TEM confirmed the presence of the $\mathrm{Ln}(\mathrm{Ln}=\mathrm{Pr}, \mathrm{Tm})$ dopant in the expected proportion. The results of quantitative EDX analysis are presented in table 3.

For doped samples the atomic concentration ratio of $\mathrm{Ga}^{3+} / \mathrm{Gd}^{3+}$ ions is increased (1.62) compared to that observed in undoped $\mathrm{Gd}_{3} \mathrm{Ga}_{5} \mathrm{O}_{12}$ (1.44) (see table 3). This is another indication that $\mathrm{Pr}^{3+}$ and $\mathrm{Tm}^{3+}$ indeed entered the structure, mainly substituting $\mathrm{Gd}^{3+}$ ions. On the other hand, the
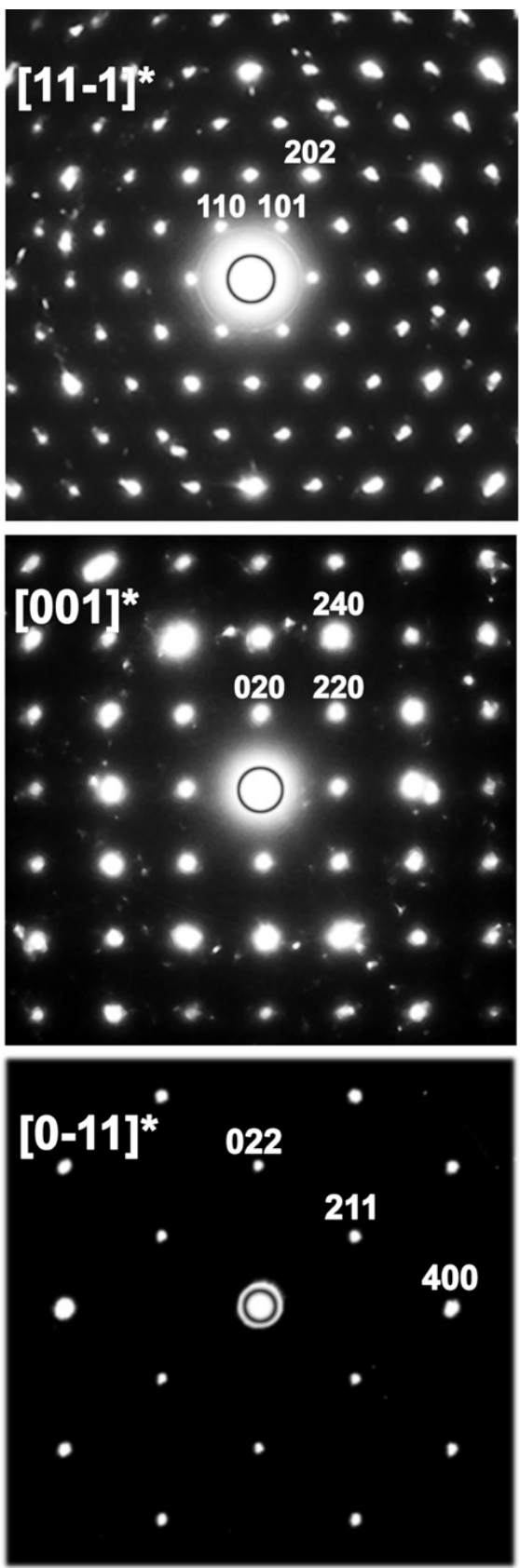

Figure 4. ED patterns of the undoped $\mathrm{Gd}_{3} \mathrm{Ga}_{5} \mathrm{O}_{12}$ along the [001]*, [011 $]^{*}$ and $[11 \overline{1}]^{*}$ zone axes.

concentration ratio $\mathrm{Gd}^{3+} / \mathrm{Ln}^{3+}(\mathrm{Ln}=\mathrm{Pr}, \mathrm{Tm})$ is quite stable and close to the ideal value of 9 . This can be used as a quantitative confirmation that a solid solution with the stoichiometry of $\mathrm{Gd}_{2.7} \mathrm{Ln}_{0.3} \mathrm{Ga}_{5} \mathrm{O}_{12}$ and a uniform dopant distribution was effectively obtained.

\subsection{Luminescence}

The room-temperature luminescence spectrum for the $1 \%$ $\mathrm{Tm}^{3+}$-doped GGG nanocrystalline sample was obtained under direct excitation in the ${ }^{1} \mathrm{D}_{2}$ level of $\mathrm{Tm}^{3+}$ ion with $355 \mathrm{~nm}$ radiation (figure 6). The luminescence spectrum is dominated by strong emission bands located around $460 \mathrm{~nm}$, which can be assigned to transitions between the Stark components of 


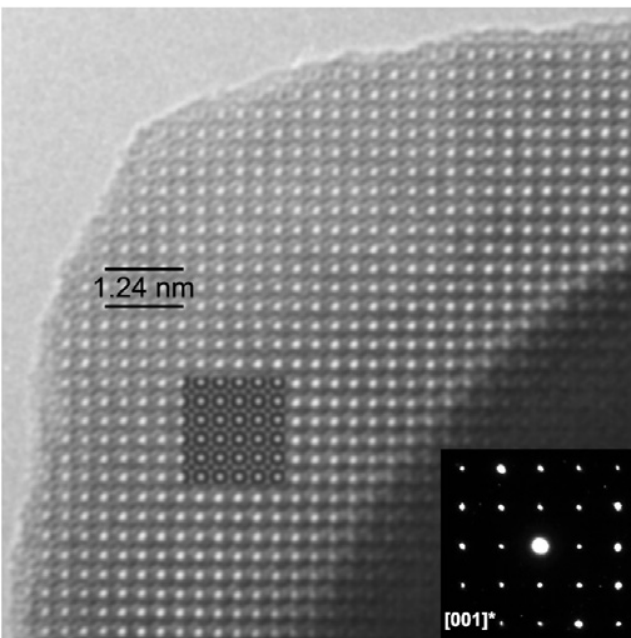

Figure 5. HREM image and corresponding ED pattern for $\mathrm{Pr}^{3+}$-doped $\mathrm{Gd}_{3} \mathrm{Ga}_{5} \mathrm{O}_{12}$ along the [001]* zone. The simulated image for a focus value of $80 \mathrm{~nm}$ and a thickness of $2.5 \mathrm{~nm}$ is shown as an inset.

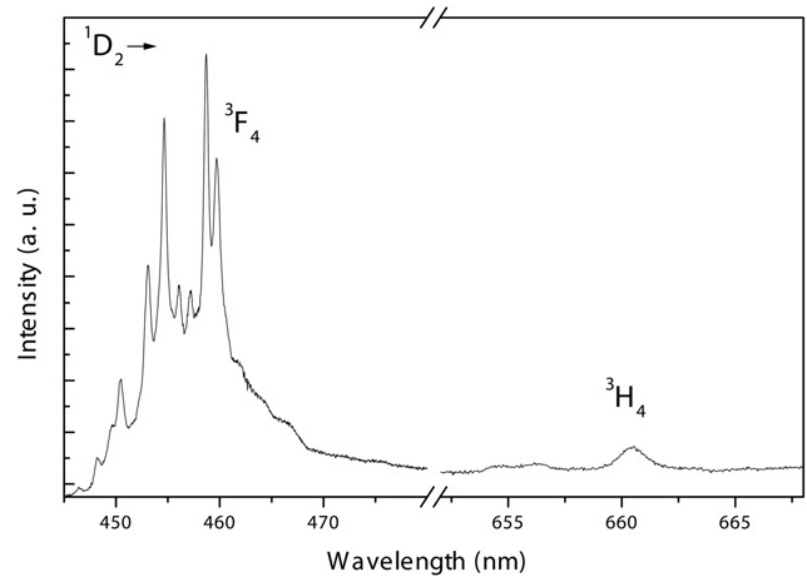

Figure 6. Room-temperature luminescence spectrum of the $1 \%$ $\mathrm{Tm}^{3+}$-doped GGG sample $\left(\lambda_{\mathrm{exc}}=355 \mathrm{~nm}\right)$.

the ${ }^{1} \mathrm{D}_{2}$ and ${ }^{3} \mathrm{~F}_{4}$ energy levels of the $\mathrm{Tm}^{3+}$ ion [43, 44]. From figure 6 it is evident that the transitions are not fully resolved due to broadening of the emission bands which overlap each other. Luminescence bands due to the ${ }^{1} \mathrm{D}_{2} \rightarrow{ }^{3} \mathrm{~F}_{4}$ transition were already observed for $\mathrm{Tm}^{3+}$-doped GGG single crystals [44] and for other garnets, such as yttrium scandium aluminium garnet (YSAG), lutetium aluminium garnet (LuAG) or yttrium aluminium garnet (YAG) [45, 46]. A weaker emission band is also observed at about $660 \mathrm{~nm}$, which can be assigned to the ${ }^{1} \mathrm{D}_{2} \rightarrow{ }^{3} \mathrm{H}_{4}$ transition [44]. Very weak features presumably due to the ${ }^{3} \mathrm{H}_{4} \rightarrow{ }^{3} \mathrm{H}_{6}$ transition are observed around $800 \mathrm{~nm}$ (not shown).

The room-temperature luminescence decay curves for the 1,5 and $10 \% \mathrm{Tm}^{3+}$-doped nanocrystalline samples are shown in figure 7 . The decay curves show a non-exponential behaviour for all the $\mathrm{Tm}^{3+}$-doped GGG samples. As evidenced from the X-ray diffraction patterns, two slightly different garnet phases have been found for the $\mathrm{Tm}^{3+}$-doped GGG samples under investigation. Therefore, the non-exponential behaviour

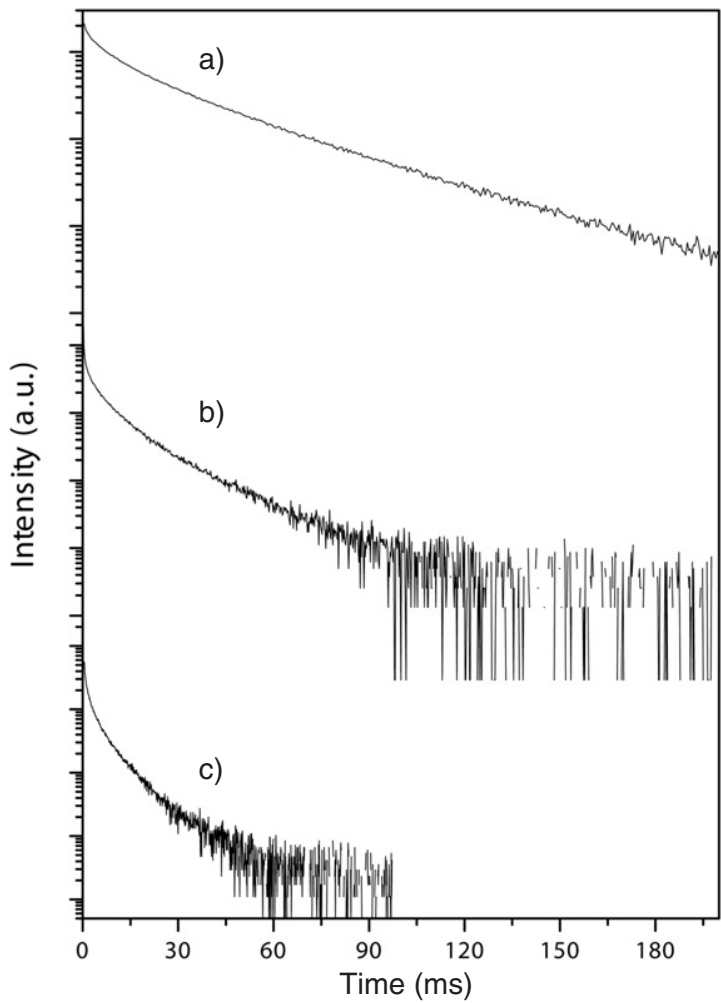

Figure 7. Room-temperature luminescence decay curves for the $1 \%$ (a), $5 \%$ (b), and $10 \%$ (c) $\mathrm{Tm}^{3+}$-doped GGG samples.

of the luminescence decays could be due to the presence of more than one emitting $\mathrm{Tm}^{3+}$ sites, accommodated in the two above-mentioned garnet phases. On the other hand, a different explanation could also be invoked to account for the nonexponential behaviour. Due to the nanocrystalline nature of the samples, a relevant percentage of the dopant $\mathrm{Tm}^{3+}$ lanthanide ions lies on the surface of the nanoparticle. The crystal field around dopant ions located on or near the surface could be different from that existing around dopant ions in the bulk of the nanoparticle. This could give rise to different transition probabilities resulting in a non-exponential behaviour of the emission decay curves. Besides, it is evident from figure 7 that the luminescence decays shorten continuously on passing from the $1 \%$ to the $5 \%$ and to the most concentrated $10 \% \mathrm{Tm}^{3+}$ doped GGG samples. From the experimental data, we have calculated the effective decay time $\tau_{\text {eff }}$ using the equation [47]

$$
\tau_{\text {eff }}=\frac{\int_{0}^{\infty} t I(t) \mathrm{d} t}{\int_{0}^{\infty} I(t) \mathrm{d} t},
$$

where $I(t)$ represents the luminescence intensity at time $t$ corrected for the background and the integrals are evaluated in a range $0<t<t_{\max }$, where $t_{\max } \gg \tau_{\text {eff. }}$. The obtained

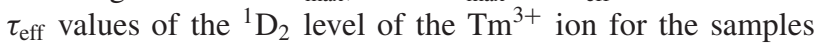
under investigation are reported in table 4 . It is clearly evident that the effective decay time decreases on increasing the dopant concentration in the GGG host. This behaviour strongly suggests that a non-radiative cross-relaxation (CR) process between the $\mathrm{Tm}^{3+}$ ions is present. Taking into account the energy levels of the $\mathrm{Tm}^{3+}$ ion in the GGG single 
Table 4. Room-temperature effective decay times $\tau_{\text {eff }}$ of the ${ }^{1} \mathrm{D}_{2}$ level of $\mathrm{Tm}^{3+}$ in doped GGG nanocrystalline samples $\left(\tau_{\text {exc }}=355 \mathrm{~nm}\right)$.

\begin{tabular}{cc}
\hline Nanocrystalline GGG:Tm & $3+$ \\
\hline 1 & $\tau_{\text {eff }}(\mu \mathrm{s})$ \\
5 & 30.9 \\
10 & 10.8 \\
& 6.5 \\
\hline
\end{tabular}

crystal [44] the most likely CR mechanism is $\left({ }^{1} \mathrm{D}_{2},{ }^{3} \mathrm{H}_{6}\right) \rightarrow$ $\left({ }^{3} \mathrm{H}_{4},{ }^{3} \mathrm{~F}_{2}\right)$. This process is non-resonant, but the mismatch (about $350 \mathrm{~cm}^{-1}$ ) can be easily bridged by the emission of one phonon. In fact, the Raman spectrum of an undoped nanocrystalline GGG sample shows a strong band peaked at a Raman shift of about $350 \mathrm{~cm}^{-1}$ [38]. The CR process of course becomes more efficient in the most concentrated sample, which also shows the shorter $\tau_{\text {eff }}$ value. In the $5 \%$ and $10 \%$ samples, the relaxation of the ${ }^{1} \mathrm{D}_{2}$ level is also enhanced by energy migration among the donor levels [48]. It is important to remark that cross-relaxation processes causing the quenching of different excited states have been observed for $\mathrm{Tm}^{3+}$-doped GGG single crystals [49], even in more diluted samples $(0.5 \%$ doping level).

The Judd-Ofelt parameters for the $\mathrm{Tm}^{3+}$ ion in the GGG host were obtained by Brenier et al [50] and turned out to be $\Omega_{2}=1.85 \times 10^{-21} \mathrm{~cm}^{2}, \Omega_{4}=9.61 \times 10^{-21} \mathrm{~cm}^{2}, \Omega_{6}=$ $7.36 \times 10^{-21} \mathrm{~cm}^{2}$. The average refractive index of GGG is 1.965 [51]. Using the Judd-Ofelt parameters and this value, the radiative lifetime $\tau_{\text {rad }}$ for the ${ }^{1} \mathrm{D}_{2}$ level results to be $75 \mu \mathrm{s}$. The percentage quantum efficiency $\eta \%$ of the ${ }^{1} \mathrm{D}_{2} \rightarrow{ }^{3} \mathrm{~F}_{4}$ emission, obtained with the formula

$$
\eta \%=100 \tau_{\text {eff }} / \tau_{\text {rad }},
$$

is 44, 15 and 9 for the 1,5 and $10 \% \mathrm{Tm}^{3+}$-doped nanocrystalline GGG samples, respectively. It is worth noting that the energy gap between the ${ }^{1} \mathrm{D}_{2}$ and the next lower lying ${ }^{1} \mathrm{G}_{4}$ level is approximately $6000 \mathrm{~cm}^{-1}$. Considering that the phonon energy cutoff for the GGG host is about $600 \mathrm{~cm}^{-1}$ [26], approximately 10 phonons would be required to bridge the energy gap between the ${ }^{1} \mathrm{D}_{2}$ and the ${ }^{1} \mathrm{G}_{4}$ levels. For this reason, the multiphonon transition probability from the ${ }^{1} \mathrm{D}_{2}$ level is very low and therefore the ${ }^{1} \mathrm{D}_{2}$ level can be depopulated by photon emission or via cross-relaxation processes. Since the effective decay times for all the nanocrystalline samples are significantly lower than the radiative lifetime, we can reasonably conclude that cross-relaxation processes are present even for the most diluted nanocrystalline sample, contributing to the non-exponential decay behaviour.

\section{Conclusions}

This work presents a structural and morphological investigation of nanocrystalline $\mathrm{Gd}_{3} \mathrm{Ga}_{5} \mathrm{O}_{12}$ samples, undoped and doped with $\mathrm{Pr}^{3+}$ or $\mathrm{Tm}^{3+}$ rare earth ions. The analysis by the Rietveld method of XRD patterns of GGG powders (undoped and doped with $\mathrm{Pr}^{3+}$ or $\mathrm{Tm}^{3+}$ ) reveals the existence of two garnet-type phases. The better agreement between the observed and the calculated patterns was found for a model with a cubic (space group $I a \overline{3} d$, usual GGG structure) and a garnet distorted orthorhombic structure. Using electron diffraction we did not find any evidence for an orthorhombic phase and we therefore conclude that the material consists of two cubic phases with a slightly different lattice parameter. One might argue though that the orthorhombic phase is a non-equilibrium phase formed during propellant synthesis and that under the electron beam this phase is transformed into a cubic phase. We have tried to avoid transformation by working under minimum illumination conditions, but we could never find evidence for the orthorhombic phase. We detected a change of cell parameter with lanthanide doping due to the difference in size between the dopant and the host ions. The volume of the unit cell of all phases increases for the Pr-doped sample and decreases for the Tm-doped sample. A similar dependence on the doping is observed for the unit-cell parameter of the cubic phase. This was a first confirmation that the dopants enter into the GGG structure.

The electron microscopy technique directly images the powder agglomerates and shows that they are constituted of aggregated single-crystal particles with an irregular shape and a size distribution ranging from 30 to $100 \mathrm{~nm}$. HREM images, for undoped and doped samples, show a stable, wellordered structure of $\mathrm{Gd}_{3} \mathrm{Ga}_{5} \mathrm{O}_{12}$ crystals without any defect or cation ordering. The presence of the dopants is confirmed by EDX microanalysis. The observed HREM images and ED patterns are in good agreement with the simulated ones for the structural model where dopant atoms are randomly distributed on the $\mathrm{Gd}^{3+}$ atomic positions. The results for the $\mathrm{Pr}^{3+}$-doped nanocrystalline GGG agree with the results obtained from EXAFS spectroscopy on the same material [52]. As a conclusion, we have found that the solution propellant method is able to produce very good solid solutions of $\mathrm{Gd}_{3} \mathrm{Ga}_{5} \mathrm{O}_{12}$, in nanocrystalline form, with a homogeneous distribution of the lanthanide dopants. Results of quantitative EDX analysis show that a solid solution with stoichiometry $\mathrm{Gd}_{\approx 2.7} \mathrm{Ln} \approx 0.3 \mathrm{Ga}_{5} \mathrm{O}_{12}(\mathrm{Ln}=$ Pr or $\mathrm{Tm})$ was effectively obtained for 10 mol\% praseodymium and thulium-doped $\mathrm{Gd}_{3} \mathrm{Ga}_{5} \mathrm{O}_{12}$ samples.

The luminescence spectrum of the $1 \% \mathrm{Tm}^{3+}$-doped nanocrystalline GGG sample is dominated by a strong emission in the blue region around $460 \mathrm{~nm}$ after excitation in the ultraviolet (at $355 \mathrm{~nm}$ ). The emission decay curves for the $\mathrm{Tm}^{3+}$-doped GGG samples show a non-exponential behaviour which can be explained by the presence of more than one phase in which the $\mathrm{Tm}^{3+}$ is accommodated, by the presence of cross-relaxation processes, and by surface effects. The presence of these processes decreases the effective decay time of the ${ }^{1} \mathrm{D}_{2}$ energy level and the quantum efficiency of the ${ }^{1} \mathrm{D}_{2} \rightarrow{ }^{3} \mathrm{~F}_{4}$ emission of the $\mathrm{Tm}^{3+}$ ion on increasing the dopant concentration in the samples under investigation. On the other hand, the quantum efficiency of the ${ }^{1} \mathrm{D}_{2} \rightarrow{ }^{3} \mathrm{~F}_{4}$ emission for the most diluted sample is relatively high (around 50\%), suggesting that the present $\mathrm{Tm}^{3+}$-doped GGG materials could be promising as blue luminophors for applications in which nanocrystalline light-emitting materials are required.

\section{Acknowledgments}

This work was performed within the framework of the Belgian IAP5-01 project and the 2003 PRIN/Cofin contract of the 
Italian Ministry for University and Research (MURST). Dr Daniele Falcomer from the University of Verona is gratefully acknowledged for his help in measuring the luminescence spectrum and emission decay curves.

\section{References}

[1] Abrahams S C and Geller S 1958 Acta Crystallogr. 11437

[2] Aubry A, Dusausoy Y, Laffaille A and Protas J 1969 Bull. Soc. Fr. Miner. Cristallogr. 92126

[3] Takeuchi Y, Haga N, Umizu S and Sato G 1982 Z. Kristallogr. 15853

[4] Eppler W R and Kryder M H 1995 J. Phys. Chem. Solids 561479

[5] Aichele T, Lorenz A, Hergt R and Görnert P 2003 Cryst. Res. Technol. 38575

[6] Ibrahim N B, Edwards C and Palmer S B 2000 J. Magn. Magn. Mater. 220183

[7] Chénais S, Druon F, Balembois F, Georges P, Brenier A and Boulon G 2003 Opt. Mater. 2299

[8] Kir'yanov A V, Aboites V, Belovolov A M, Timoshechkin M I, Belovolov M I, Damzen M J and Minassian A 2002 Opt. Express 10832

[9] McKittrick J, Shea L E, Bacalski C F and Bosze E J 1999 Displays 19169

[10] Talin A A, Dean K A and Jaskie J E 2001 Solid State Electron. 45963

[11] Hunt C E and Chakhovskoi A G 1997 J. Vac. Sci. Technol. B 15516

[12] Fukaya S, Adachi K, Obara M and Kumagai H 2001 Opt. Commun. 187373

[13] Ye T, Guiwen Z, Weiping Z and Shangda X 1997 Mater. Res. Bull. 32501

[14] Tessari G, Bettinelli M, Speghini A, Ajò D, Pozza G, Depero L E, Allieri B and Sangaletti L 1999 Appl. Surf. Sci. 144/145 686

[15] Shikao S and Jiye W 2001 J. Alloys Compounds 32782

[16] Ramanathan S, Kakade M B, Roy S K and Kutty K K 2003 Ceram. Int. 29477

[17] Ekambaram S and Patil K C 1995 J. Alloys Compounds 217104

[18] Hreniak D and Strek W 2002 J. Alloys Compounds 341183

[19] Qiu F, Pu X, Li J, Liu X, Pan Y and Guo J 2005 Ceram. Int. 31663

[20] Sharma P K, Jilavi M H, Schmidt H and Varadan V K 2000 Int. J. Inorg. Mater. 2407

[21] Zhao G, Li T, He X and Xu J 2002 Mater. Lett. 561098

[22] Bazzoni M, Bettinelli M, Daldosso M, Enzo S, Serra F and Speghini A 2005 J. Solid State Chem. 1782301

[23] Wyss Chr P et al 1999 J. Lumin. 82137

[24] Brenier A, Courrol L C, Madej C and Boulon G 1994 Phys. Rev. B 49881

[25] Carazo-Salas R, Murday R, Proulx P P, Karbo P, Cormier G and Capobianco J A 1996 Opt. Mater. 6203
[26] Vetrone F, Boyer J C, Capobianco J A, Speghini A and Bettinelli M 2003 J. Phys. Chem. B 10710747

[27] Boyer J C, Vetrone F, Capobianco J A, Speghini A, Zambelli M and Bettinelli M 2004 J. Lumin. 106263

[28] Naccache R, Vetrone F, Boyer J C, Capobianco J A, Speghini A and Bettinelli M 2004 J. Nanosci. Nanotechnol. 41025

[29] Boyer J C, Vetrone F, Capobianco J A, Speghini A and Bettinelli M 2004 Chem. Phys. Lett. 390403

[30] Mita Y, Togashi M and Yamamoto H 2000 J. Lumin. 87-89 1026

[31] Sharma P K, Jilavi M H, Nass R and Schmidt H 1999 J. Lumin. 82187

[32] Lopez O A, McKittrick J and Shea L E 1997 J. Lumin. 71

[33] Williams K D, Yuan H and Tissue M B 1999 J. Lumin. 83/84 297

[34] Allieri B, Depero L E, Marino A, Sangaletti L, Caporaso L, Speghini A and Bettinelli M 2000 Mater. Chem. Phys. 66164

[35] Vaqueiro P and López-Quintela M A 1998 J. Mater. Chem. 8161

[36] Krsmanović R, Polizzi S and Canton P 2005 Mater. Sci. Forum 494143

[37] Maglia F, Buscaglia V, Gennari S, Ghigna P, Dapiaggi M, Speghini A and Bettinelli M 2006 J. Phys. Chem. B 1106561

[38] Naccache R, Vetrone F, Capobianco J A, Speghini A and Bettinelli M 2007 in preparation

[39] Rietveld H M 1969 J. Appl. Crystallogr. 265

[40] Izumi F 1993 The Rietveld Method (New York: Oxford University Press) chapter 13

[41] Kim Y I and Izumi F 1994 J. Ceram. Soc. Japan 102401

[42] Dukhovskaya E L, Saksonov Y G and Titova A G 1973 Neorg. Mater. 9809

Sawada H 1997 J. Solid State Chem. 132300

[43] Brenier A, Garapon C, Madej C, Pédrini C and Boulon G 1994 J. Lumin. 62147

[44] Lupei A, Lupei V, Grecu S, Tiseanu C and Boulon G 1994 J. Appl. Phys. $\mathbf{7 5} 4652$

[45] Gruber J B, Seltzer M D, Hills M E, Stevens S B and Morrison C A 1993 J. Appl. Phys. 731929

[46] Cicillini S A, Pires A M and Serra O A 2004 J. Alloys Compounds 374169

[47] Nakazawa E 1999 Phosphor Handbook (Boca Raton, FL: CRC Press) p 104

[48] Martín I R, Rodríguez V D, Alcalá R and Cases R 1993 J. Non-Cryst. Solids 161294

[49] Brenier A, Madej C, Pédrini C and Boulon G 1991 J. Phys.: Condens. Matter 3203

[50] Brenier A, Courrol L C, Pédrini C, Madej C and Boulon G 1994 Phys. Rev. B 49881

[51] Gill D S, Anderson A A, Eason R W, Warburton T J and Shepherd D P 1996 Appl. Phys. Lett. 6910

[52] Daldosso M, Falcomer D, Speghini A, Ghigna P and Bettinelli M 2007 Opt. Mater. at press (doi:10.1016/j.optmat.2007.05.042)

[53] Shannon R D 1976 Acta Crystallogr. A 32751 